\title{
Review: aspirin lowers the risk for MI but not for stroke in people who do not have established vascular disease
}

Hart $R G$, Halperin JL, McBride $R$, et al. Aspirin for the primary prevention of stroke and other major vascular events. Meta-analysis and hypotheses. Arch Neurol 2000 Mar;57:326-32.

QUESTION: For people who do not have clinically recognised vascular disease, is aspirin effective for reducing stroke and other vascular events?

\section{Data sources}

Studies were identified by searching for English language articles in $\{$ Medline $\} *$ (1980-98) with the terms clinical trial, aspirin, and stroke; the Cochrane Collaboration Trials Registry; the Antiplatelet Trialists' Collaboration lists of publications; and bibliographies of review articles.

\section{Study selection}

Studies were selected if they were randomised controlled trials or large observational studies of aspirin in any dose; trials compared aspirin with placebo for primary prevention of stroke; data were provided on people who did not have established vascular disease at baseline; and outcomes were stroke, myocardial infarction, or other major vascular events.

\section{Data extraction}

Data were extracted on study population and size, aspirin dose, and outcomes.

\section{Main results}

5 randomised controlled trials met the inclusion criteria. 52251 participants (mean age 57 y, 80\% men) were studied, and mean follow up was 4.6 years. Aspirin and placebo doses were $75 \mathrm{mg} /$ day for 21330 participants, $325 \mathrm{mg}$ every other day for 22071 participants, and 500 to $650 \mathrm{mg}$ /day for 8850 participants. Aspirin was associated with a small decreased risk for myocardial infarction and a trend toward decreased all cause mortality, but it was not associated with decreased risk for stroke, haemorrhagic stroke, or vascular death (table). The participants' underlying vascular risk or the dose of aspirin used was not related to any outcome.

Aspirin v placebo for primary prevention of vascular events at mean follow up of 4.6 years $\dagger$

\begin{tabular}{lllll} 
Outcomes & $\begin{array}{l}\text { Number } \\
\text { of studies }\end{array}$ & Patients & $\begin{array}{l}\text { Relative risk } \\
(95 \% \mathrm{Cl})\end{array}$ & NNT (Cl) \\
Myocardial infarction & 5 & All & $0.74(0.68$ to 0.82$)$ & $115(91$ to 164$)$ \\
\hline All cause mortality & 5 & All & $0.94(0.87$ to 1.01$)$ & Not significant \\
\hline Stroke & 5 & All & $1.08(0.95$ to 1.24$)$ & Not significant \\
\hline Haemorrhagic stroke & 4 & All & $1.35(0.88$ to 2.10$)$ & Not significant \\
\hline Vascular death & 5 & All & $0.93(0.93$ to 1.03$)$ & Not significant \\
\hline
\end{tabular}

$\dagger$ Abbreviations defined in glossary: NNT and $\mathrm{Cl}$ calculated from data provided by author.

\section{Conclusion}

In participants who have no clinical evidence of vascular disease, aspirin reduces the risk for myocardial infarction but does not reduce the risk for stroke or vascular death.

*Information provided by author.

\section{COMMENTARY}

According to the analysis by the Antiplatelet Trialists Collaboration, ${ }^{1}$ aspirin is effective for the secondary prevention of stroke. Although physicians are often asked whether aspirin should be taken, it remains unclear whether it is beneficial for the primary prevention of stroke or other vascular events. Only 2 large prospective trials of aspirin in healthy men have been completed. Data from the ongoing Women's Health Study will soon provide evidence on the efficacy of aspirin for primary prevention of heart disease and stroke among healthy women. ${ }^{2}$

In this methodologically sound meta-analysis of the 5 existing randomised trials on the subject, Hart et al conclude that aspirin is not beneficial in the primary prevention of stroke among people with no vascular disease. Indeed, aspirin may increase the risk for stroke, although this trend is not significant. Results of the Physicians' Health Study, ${ }^{3}$ that aspirin treatment may prevent myocardial infarction among men $>55$ years of age who have risk factors for cardiovascular disease, are supported by this systematic review. A nonsignificant trend showed a higher rate of intracranial haemorrhage among people who use aspirin. Whether intracranial haemorrhage explains the higher stroke incidence with aspirin use in primary stroke prevention studies is unclear, but it is a cause for concern. Aspirin should therefore be prescribed for stroke prevention only for patients with vascular disease, particularly those with a history of transient ischaemic attack or stroke. For patients who cannot tolerate aspirin, clopidogrel or ticlopidine are effective alternatives. Richard L Harvey, MD Northwestern University Medical School Chicago, Illinois, USA

1 Antiplatelet Trialists' Collaboration. Collaborative overview of randomised trials of antiplatelet therapy-I: prevention of death, myocardial infarction, and stroke by prolonged antiplatelet therapy in various categories of patients. BMJ 1994;308:81-106

2 Buring JE, Hennekens CH. The women's health study: summary of the study design. J Myocardial Ischemia 1992;4:27-9.

3 Steering Committee of the Physicians' Health Study Research Group. Final report on the aspirin component of the ongoing Physicians' Health Study. N EnglJ Med 1989;321:129-35. 